\title{
Polymorphism on Chromosome 9p21.3 Is Associated with Severity and Early-Onset CAD in Type 2 Diabetic Tunisian Population
}

\author{
Kaouthar Abid, Donia Mili, and Abderraouf Kenani \\ Laboratory of Biochemistry, UR 12ES08, Faculty of Medicine, 5000 Monastir, Tunisia \\ Correspondence should be addressed to Kaouthar Abid; kaouthar_abid@yahoo.fr
}

Received 7 July 2015; Revised 13 August 2015; Accepted 23 August 2015

Academic Editor: Fabrizia Bamonti

Copyright (C) 2015 Kaouthar Abid et al. This is an open access article distributed under the Creative Commons Attribution License, which permits unrestricted use, distribution, and reproduction in any medium, provided the original work is properly cited.

\begin{abstract}
Multiple association studies found that the human 9p21.3 chromosome locus is a risk factor for atherosclerosis. The purpose of this study was to investigate the association of the severity and early-onset of coronary artery disease with variant rs1333049 on chromosome 9p21.3 polymorphism and the impact of this variant on cardiovascular risk factors in type 2 diabetic patients. The study population consisted of a control CAD group (101 patients) and 273 consecutive type 2 diabetic patients. Severity and extent of coronary atherosclerosis were scored numerically using the Gensini scoring system. The diabetic population was divided into three groups according to Gensini score: Group 1: no stenosis; Group 2: moderate CAD; Group 3, severe CAD. The homozygous CC genotype of rs1333049 was significantly associated with CAD in Group 2 (OR: 1.36; $p=0.02$ ) and Group 3 (OR: 5.77, $p<0.001$ ) compared to Group 1 (OR: $0.18 ; p=0.2$ ) and control group (OR: 0.22; $p=0.21$ ). Among diabetic patients with early-onset CAD, CC genotype carriers had significantly higher Gensini scores than non-CC genotype carriers $(49 \pm 21.3$ versus $14.87 \pm 25.22$; $p<0.001)$. The homozygous CC genotype of rs1333049 confers a magnified risk of early-onset and severe CAD in type 2 diabetic Tunisian population.
\end{abstract}

\section{Introduction}

The association of genetics and environmental factors (such as life-style) plays a major role in the pathogenesis of coronary artery disease (CAD) and type 2 diabetes (T2D). The genetic architecture of CAD and that of T2D are dependent on multiple genetic and environmental triggers that may be specific to each disease. The chromosome $9 \mathrm{p} 21.3$ has been identified as the locus with strongest association with coronary artery disease in multiple independent large-scale genome-wide association studies [1-3].

It is the most consistently replicated genetic locus for CAD $[1,4]$ and T2D $[5,6]$. Recent genome-wide scanning has implicated chromosome 9p21.3 as a locus conferring susceptibility to CAD [2,7-9]. This increased risk is independent of traditional risk factors, including gender, age, obesity, smoking, hypertension, and hyperlipidemia [5]. Since rs1333049 polymorphism on chromosome 9p21.3 has a strong association with CAD [10], we hypothesized that such SNP on chromosome 9p21.3 may have an amplifying impact on early-onset and severity of CAD. This is the first study evaluating the association between $9 \mathrm{p} 21.3$ and CAD in Tunisian population. The purpose of the present study was to investigate the relationship between one SNP ( $\sin$ gle nucleotide polymorphism), rs1333049, on chromosome 9p21.3 and susceptibility to CAD, the effect of this SNP on cardiovascular risk factors, severity, and early-onset of CAD in type 2 diabetic Tunisian population.

\section{Methods}

2.1. Patients. The study population consisted of a total number of 374 patients composed of a control CAD group of 101 nondiabetic angiographic control cohort with CAD and 273 consecutive type 2 diabetic patients undergoing a routine coronary angiography to evaluate suspected CAD. Subjects were defined with coronary artery disease (CAD) when presenting a stenosis $>50 \%$ in at least one major coronary 
artery. Subjects were defined without coronary artery disease (no CAD) when presenting a stenosis $<50$ in at least one major coronary artery. The severity and the extent of coronary artery disease were evaluated with the Gensini score [11]. The Gensini score was computed by assigning a severity score to each coronary stenosis according to the degree of luminal narrowing and its geographic importance. The study population was divided into three groups: Group 1: patients with no stenosis (Gensini score $=0$ ); Group 2: patients with moderate CAD (Gensini score < 40); Group 3: patients with severe CAD (Gensini score > 40). Hypertension was diagnosed as blood pressure of higher than $140 / 90 \mathrm{mmHg}$, which was measured according to guidelines [12] and/or the current use of antihypertensive drugs. Diabetic subjects were defined by a fasting plasma glucose $>7.0 \mathrm{mmol} / \mathrm{L}$ or by the use of antidiabetic drugs [13]. Hyperlipidemia was defined by either high cholesterol levels $(\mathrm{TC}>5.17 \mathrm{mmol} / \mathrm{L}$ ) or high triglycerides levels (TG $>1.70 \mathrm{mmol} / \mathrm{L}$ ) or both or by the use of hypocholesterolemic drugs. Obese subjects were defined by a BMI $>30.0 \mathrm{Kg} / \mathrm{m}^{2}$. Data on age, sex, smoking, and smoking history were collected from the participants' medical records or by direct interviews. Fasting concentrations of TC, TG, LDL-C, and HDL-C were measured using standard methods [14]. Patients were diagnosed with hyperlipidemia if they had serum levels of total cholesterol (TC) $>5.7 \mathrm{mmol} / \mathrm{L}$, triglycerides (TG) $>1.7 \mathrm{mmol} / \mathrm{L}$, lowdensity lipoprotein cholesterol (LDL-C) $>3.64 \mathrm{mmol} / \mathrm{L}$, or high-density lipoprotein cholesterol (HDL-C) $<0.91 \mathrm{mmol} / \mathrm{L}$. Early-onset CAD was defined as clinical CAD occurring by age $\leq 55$ years in male or $\leq 60$ years in female patients $[15,16]$. This study was approved by our hospital ethical committee. All participants were of Tunisian origin and gave their informed consent for this study.

2.2. Biochemical Analysis. Blood samples were taken for biochemical analysis following overnight fasting. CT, TG, and HDL-C concentrations were determined at accredited clinical laboratories using routine clinical methods. LDL-C concentrations were calculated using the Friedewald equation [17].

2.3. DNA Analysis. Genomic DNA was prepared from white blood cells using the salting-out method [18]. Genotypes for the variant 9 p21.3 were determined by polymerase chain reaction (PCR).

2.4. Genotyping. Allele specific primers for the ancestral (C) and derived $(\mathrm{G})$ alleles of rs1333049: $\mathrm{C}>\mathrm{G}$, were designed to selectively amplify the relevant target sequences [19]. The sequence of the forward primer for the $\mathrm{C}$ allele was $5^{\prime}$ TCC TCA TAC TAA CCA TAT GAT CAA CAG TTC-3' and for $G$ allele the sequence was $5^{\prime}$-TCC TCA TAC TAA CCA TAT GAT CAA CAG TTG-3'. The internal control primer sequence was $5^{\prime}$-GAA GAT CAT ACC CGA AGT AGA GCT GC-3'. For all the forward primers a common reverse primer was used, with the sequence $5^{\prime}$-ATA CCA CAG TGA ACA TAA TTG TGC ATA CAT-3' . Amplification of ancestral and derived alleles was performed separately in a total reaction volume of $20 \mu \mathrm{L}$ each, containing $0.2 \mu \mathrm{M}$ deoxynucleotide triphosphates, $1 x$ Taq buffer, $3 \mathrm{mM} \mathrm{MgCl}_{2}$, $0.2 \mathrm{Mm}$ allele specific forward primer, $0.3 \mu \mathrm{M}$ reverse primer, $0.1 \mu \mathrm{M}$ internal control primer, and 1.0 U Taq polymerase. Thermocycling consisted of an initial denaturation step of $94^{\circ} \mathrm{C}$ for $4 \mathrm{~min}$ followed by 35 cycles of $94^{\circ} \mathrm{C}$ for $1 \mathrm{mn} \mathrm{s}$, $61^{\circ} \mathrm{C}$ for $30 \mathrm{~s}$, and $72^{\circ} \mathrm{C}$ for $40 \mathrm{~s}$ and a final extension cycle of $72^{\circ} \mathrm{C}$ for $7 \mathrm{~min}$. The amplified products consisting of a $280 \mathrm{bp}$ fragment for the allele specific primers and $500 \mathrm{bp}$ for the internal control were electrophoretically separated on $2 \%$ agarose gels, and DNA bands were visualized by UV transillumination, the image was documented using the BioCap MW software (ver. 11.01, Vilber Lourmat, France) and the genotype data were then calculated.

2.5. Statistical Analyses. All statistical analysis was performed using version 11.0 of the Statistical Package for the Social Sciences: SPSS (SPSS Inc., Chicago, Illinois, USA). Continuous variables are presented as mean \pm standard deviation, and categorical data are summarized as frequencies or percentages. Normal distribution of continuous variables was evaluated with the Kolmogorov-Smirnov test, and differences among groups were analyzed by one-way analysis of variance (ANOVA). For categorical variables, differences between groups were evaluated by the chi-square test. Odds ratios (ORs) of CAD for CC genotype on rs1333049, and other risk factors, were estimated by multivariate logistic regression analyses. The significance of multiplicative interactions between CC genotype and covariates was determined by a logistic regression model. The relationship between genotype and coronary disease was evaluated with the Mann-Whitney $U$ test. A 2 -sided probability level of $\leq 0.05$ was considered significant.

\section{Results}

3.1. Clinical Characteristics and Genotype Frequencies. Table 1 shows clinical features and genotype frequencies of control group, diabetic patients with normal arteries, CAD patients with Gensini score $<40$, and CAD patients with Gensini score $>40$. Compared to the diabetic population, patients of the control CAD group are younger and have a decreased percentage of family history of CAD (all $p<0.05$ ). As shown, patients with no stenosis (Group 1) are younger than patients with CAD (Groups 2 and 3), all $p<0.05$. There is a significant linear increase in the percentages of subjects with a family history for CAD and in cholesterol, TG, and LDL levels (all $p<0.05$ ). No significant differences in sex, $\mathrm{HbAlc}$, and HDL-C levels were found among the subgroups (all $p>0.05$ ). The percentages of patients treated with insulin were significantly correlated with the increasing extent of the coronary atherosclerosis. There is a significant linear increase in the percentages of subjects with hyperlipidemia, obesity, and smoking history and in BMI values. The homozygous CC genotype was significantly more common in patients with both CAD and T2D groups $(p<0.05)$.

3.2. Association between rs1333049 Polymorphism, Biochemical Measurements, and Clinical Features. Biochemical measurements and clinical features with respect to various 
TABLE 1: Baseline clinical characteristics and biochemical assessments.

\begin{tabular}{|c|c|c|c|c|c|c|}
\hline & Control CAD group & Total of the diabetic population & Group 1 & Group 2 & Group 3 & $p$ \\
\hline$N$ & 101 & 273 & 138 & 36 & 100 & - \\
\hline Gender: male/female (\%) & $56,4 / 43,6$ & $52,7 / 47,3$ & $52,2 / 47,8$ & $58,3 / 41,7$ & $52 / 48$ & 0,651 \\
\hline Age (years) & $56,27 \pm 16,75$ & $62,82 \pm 12,26$ & $60,36 \pm 13,42$ & $65,92 \pm 9,17$ & $65,26 \pm 10,91$ & 0,003 \\
\hline Family history for CAD (\%) & 46,9 & 60,4 & 50,7 & 63,9 & 72 & 0,007 \\
\hline Hypertension (\%) & 40,1 & 59,3 & 47,8 & 63,9 & 75 & $<0,001$ \\
\hline BMI & $27,21 \pm 3,99$ & $29,59 \pm 4,51$ & $28,63 \pm 4,74$ & $30,03 \pm 2,02$ & $30,73 \pm 4,55$ & 0,002 \\
\hline Obesity (\%) & 30,4 & 47,3 & 35,5 & 61,1 & 58 & 0,001 \\
\hline $\mathrm{HbAlc}(\mathrm{mmol} / \mathrm{L})$ & $7,1 \pm 3,52$ & $10,52 \pm 4,20$ & $10,27 \pm 3,94$ & $10,08 \pm 4,30$ & $11,06 \pm 4,50$ & 0,251 \\
\hline Smoking (\%) & 31,9 & 42,1 & 33,3 & 58,3 & 49 & 0,006 \\
\hline Cholesterol (mmol/L) & $4,58 \pm 1,3$ & $4,72 \pm 1,12$ & $4,73 \pm 1,14$ & $4,52 \pm 0,87$ & $4,78 \pm 1,18$ & 0,414 \\
\hline $\mathrm{LDL}(\mathrm{mmol} / \mathrm{L})$ & $2,91 \pm 1,13$ & $3,73 \pm 1,45$ & $3,28 \pm 1,22$ & $3,86 \pm 1,35$ & $4,29 \pm 1,57$ & $<0,001$ \\
\hline $\mathrm{HDL}(\mathrm{mmol} / \mathrm{L})$ & $1,14 \pm 0,44$ & $1,23 \pm 0,52$ & $1,26 \pm 0,51$ & $1,17 \pm 0,40$ & $1,22 \pm 0,58$ & 0,455 \\
\hline $\mathrm{TG}(\mathrm{mmol} / \mathrm{L})$ & $1,27 \pm 0,64$ & $2,21 \pm 1,29$ & $1,76 \pm 1,06$ & $2,07 \pm 0,98$ & $2,91 \pm 1,40$ & $<0,001$ \\
\hline Hyperlipidemia (\%) & 55,6 & 49,5 & - & 97,2 & 100 & $<0,001$ \\
\hline Insulin (\%) & - & 52,4 & 36,2 & 61,1 & 72 & $<0,001$ \\
\hline \multicolumn{7}{|l|}{ Rs1333049 genotypes } \\
\hline GG (\%) & 72,3 & 25,3 & 40,6 & 11,1 & 10 & 0,377 \\
\hline GC (\%) & 22,8 & 46,2 & 52,9 & 47,2 & 36 & 0,136 \\
\hline CC (\%) & 5 & 28,6 & 6,5 & 41,7 & 54 & $<0,001$ \\
\hline
\end{tabular}

Data are number (\%) and mean \pm SD. BMI: body mass index; CAD: coronary artery disease; HbAlc: glycosylated hemoglobin; HDL-C: HDL cholesterol; LDLC: LDL cholesterol; TC: cholesterol total; TG: triglycerides.

TABLE 2: Changes in biochemical measurements and clinical characteristics among the three genotypes in the three groups of the diabetic population.

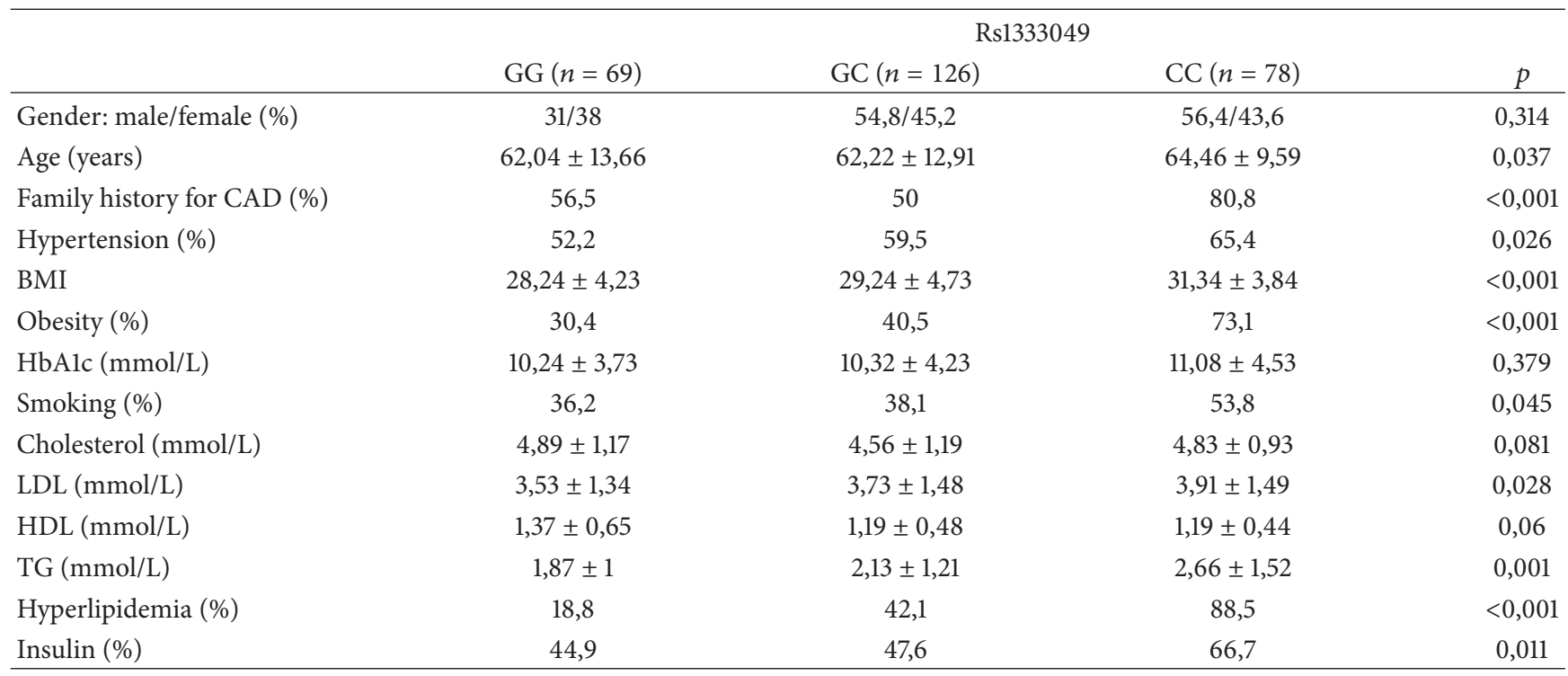

Data are number (\%) and mean \pm SD. BMI: body mass index; CAD: coronary artery disease; HbAlc: glycosylated hemoglobin; HDL-C: HDL cholesterol; LDLC: LDL cholesterol; TC: cholesterol total; TG: triglycerides.

genotypes are listed in Table 2 . Subjects with family history of CAD, hypertension, obesity, smoking, insulin, and hyperlipidemia percentages were significantly higher in CC genotype carriers (all $p<0.05$ ), likewise for BMI, LDL-C, and TG values. There were no significant differences in changes of gender, cholesterol, HDL-C, and HbAlc value among the three genotypes in the whole population (all $p>0.05$ ) (Table 2).

3.3. Association between rs1333049 Polymorphism Severity and Early-Onset of CAD. In Table 3 there was an association between $\mathrm{CC}$ genotype and severity of CAD in all patients 
TABLE 3: Association of rs1333049 CC genotype with severity and early-onset of CAD in diabetic patients.

\begin{tabular}{lccc}
\hline & \multicolumn{3}{c}{$\operatorname{Rs} 1333049$} \\
& $\mathrm{CC}(n=78)$ & Non-CC $(n=237)$ & $p$ \\
\hline Gensini score & & & \\
All patients & $49 \pm 21,3$ & $14,87 \pm 25,22$ & $<0,001$ \\
Early-onset CAD & $50,98 \pm 18,71$ & $11,34 \pm 22,80$ & $<0,001$ \\
Late-onset CAD & $45,57 \pm 25,26$ & $18 \pm 26,95$ & $<0,001$ \\
\hline
\end{tabular}

Data are mean \pm SD. CAD: coronary artery disease.

$(p<0.001)$, since Gensini scores differ significantly between CC genotype carriers and non-CC genotype carriers (49 $\pm 21,3$ versus $14,87 \pm 25,22$ ). This study also showed that diabetic CC genotype carriers with early-onset CAD had significantly higher Gensini scores than diabetic non-CC genotype carriers $(50,98 \pm 18,71$ versus $11,34 \pm 22,80$; $p<$ $0.001)$.

3.4. Multivariable Analysis. A multiple logistic regression analysis was performed with CAD severity (Gensini score $<$ or $>40)$ as the dependent variable and the following as risk factors: age, family history of CAD, hypertension, smoking, hyperlipidemia, obesity, BMI, triglycerides, LDL$\mathrm{C}$, and insulin (Table 4). Analysis showed that BMI (OR: 1.03; 95\% CI: 0.92-1.12; $p=0.03$ ), TG levels (OR: 2.4; 95\% CI: $1.82-$ 3.29; $p<0.001$ ), LDL-C levels (OR: 1.76; 95\% CI: 1.42-2.18; $p<0.001$ ), family history of CAD (OR: 1.85 ; 95\% CI: 1.013.89 ; $p=0.01$ ), hypertension (OR: 3.26; 95\% CI: 1.52-6.98; $p<0.001$ ), obesity (OR: 1.53 ; 95\% CI: 1.19-2.01; $p=0.02$ ), smoking (OR: 1.8; 95\% CI: 1.2-3.89; $p<0.001$ ), insulin (OR: 4.71; 95\% CI: $2.3-9.65 ; p<0.001$ ), and hyperlipidemia (OR: 2.56; 95\% CI: 1.12-4.06; $p<0.001)$ are important risk factors of CAD severity in type 2 diabetic patients. Further analysis showed that CC genotype was associated with CAD in Group 3 patients (OR 5.77; 95\% CI 3.29-10.12; $p<0.001$ ) and Group 2 patients (OR 1.36; 95\% CI 1.06-1.52; $p=0.02$ ) with increasing severity of CAD compared with Group 1 patients without CAD (OR 0.18; 95\% CI 0.1-0.31; $p=0.2$ ) and with the control CAD group (OR0.22; 95\%CI 0.1-0.43; $p=0.21$ ). So, CC genotype of rs1333049 increases the severity of CAD in Tunisian type 2 diabetic patients.

\section{Discussion}

The present study has examined the CAD risk factors changes according to CAD severity and the association of CC genotype of rs1333049 with the severity and early-onset of CAD among type 2 diabetic subjects. The principal finding of this study is that the severity and early-onset of CAD are related to the CC genotype of rs1333049.

Many genome-wide association studies have shown that genetic variations on chromosome 9p21.3 were associated with increased risk of diabetes and CAD in the general population $[1-3,8]$.

Interestingly, Doria et al. [20] observed that the homozygous GG genotype of rs2383206 on chromosome 9p21.3 had a greater effect on the risk of CAD in diabetic patients than CC genotype in the general population. In the present study, we found that the homozygous CC genotype of rs1333049 was associated with severity of CAD in diabetic patients. Furthermore, in our population, CC genotype carriers had significantly higher smoking, hypertension, hyperlipidemia, family history of CAD, and obesity percentages than nonCC genotype carriers; it is the same for BMI, LDL-C, and TG levels. These observations suggest that these factors are important risk factors of $\mathrm{CAD}$ for the diabetic patients in the Tunisian population.

The mechanisms of severity of CAD and T2D in patients with acute coronary syndrome (ACS) are complex and not so clear at present. While many genetic studies [21], for CAD and T2D, have identified the 9p21.3 locus as a common risk region, there are no functional variants (noncoding variants) within this region. Therefore, the relation between the 9 p21.3 locus and the genetic principles of these two diseases remains poorly understood.

The essential mechanisms by which SNPs on chromosome 9p21.3 contribute to early-onset and severity of CAD in diabetes remain unclear.

In a family-based study, Meng et al. [16] observed that chromosome 9p21.3 is associated with early-onset CAD in the Irish population. In the present large-sample study, we found that after investigating their association with traditional risk factors homozygous CC genotype carriers were found to have an increased risk for early-onset CAD in diabetic patients. Moreover, among diabetic patients with early-onset CAD, CC genotype carriers had significantly higher Gensini scores than non-CC genotype carriers. These observations suggest that there may be a common genetic basis for diabetes and CAD in a homozygous genotype of chromosome 9p21.3 [5].

\section{Conclusion}

This study demonstrated that 9p21.3 polymorphism is significantly associated with CAD in a Tunisian population, and the homozygous CC genotype of rs1333049 confers a magnified risk of early-onset and severe CAD in type 2 diabetic patients.

\section{Abbreviations}

ACS: Acute coronary syndrome

ANOVA: Analysis of variance

BMI: $\quad$ Body mass index

CAD: Coronary artery disease

DNA: Deoxyribonucleic acid

HbAlc: Glycosylated hemoglobin Alc

HDL-C: HDL cholesterol

LDL-C: LDL cholesterol

OR: Odds ratio

PCR: Polymerase chain reaction

SNP: Single nucleotide polymorphism

SPSS: $\quad$ Statistical Package for the Social Sciences

TC: $\quad$ Total cholesterol

TG: $\quad$ Triglyceride

T2D: $\quad$ Type 2 diabetes. 
TABLE 4: Multivariable logistic regression analysis of independent determinants for CAD.

\begin{tabular}{|c|c|c|c|c|c|c|c|c|c|c|c|c|}
\hline & \multicolumn{3}{|c|}{ Control CAD group } & \multicolumn{9}{|c|}{ Diabetic population } \\
\hline & \multirow[b]{2}{*}{ OR } & \multirow[b]{2}{*}{$95 \% \mathrm{CI}$} & \multirow[b]{2}{*}{$p$} & \multicolumn{3}{|c|}{ Group 1} & \multicolumn{3}{|c|}{ Group 2} & \multicolumn{3}{|c|}{ Group 3} \\
\hline & & & & OR & $95 \% \mathrm{CI}$ & $p$ & OR & $95 \% \mathrm{CI}$ & $p$ & OR & $95 \% \mathrm{CI}$ & $p$ \\
\hline rs1333049 (CC versus non-CC) & 0,22 & $0,1-0,43$ & 0,21 & 0,18 & $0,1-0,31$ & 0,2 & 1,36 & $1,06-1,52$ & 0,02 & 5,77 & $3,29-10,12$ & $<0,001$ \\
\hline Family history for CAD (\%) & 0,35 & $0,11-0,69$ & 0,12 & 0,56 & $0,27-1,16$ & 0,12 & 2,05 & $1,09-3,87$ & 0,02 & 1,85 & $1,01-3,89$ & 0,01 \\
\hline Hypertension (\%) & 0,67 & $0,22-0,98$ & 0,24 & 0,31 & $0,17-0,57$ & 0,52 & 1,28 & $1,14-1,56$ & 0,002 & 3,26 & $1,52-6,98$ & $<0,001$ \\
\hline BMI & 0,80 & $0,60-1,07$ & 0,14 & 0,97 & $0,88-1,07$ & 0,58 & 1,02 & $0,94-1,13$ & 0,04 & 1,03 & $0,92-1,12$ & 0,03 \\
\hline Obesity (\%) & 0,96 & $0,56-1,34$ & 0,09 & 1,54 & $0,58-4,12$ & 0,38 & 1,04 & $0,44-2,46$ & 0,04 & 1,53 & $1,19-2,01$ & 0,02 \\
\hline Smoking (\%) & 0,42 & $0,13-0,77$ & 0,99 & 0,52 & $0,25-1,09$ & 0,08 & 2,11 & $1,12-3,99$ & 0,02 & 1,8 & $1,2-3,89$ & $<0,001$ \\
\hline $\mathrm{LDL}(\mathrm{mmol} / \mathrm{L})$ & 0,48 & $0,12-0,83$ & 0,28 & 0,56 & $0,45-0,71$ & 0,04 & 1,64 & $1,5-1,82$ & $<0,001$ & 1,76 & $1,42-2,18$ & $<0,001$ \\
\hline $\mathrm{TG}(\mathrm{mmol} / \mathrm{L})$ & 0,21 & $0,12-0,33$ & 0,88 & 0,4 & $0,3-0,54$ & 0,07 & 1,45 & $1,33-1,61$ & $<0,001$ & 2,4 & $1,82-3,29$ & $<0,001$ \\
\hline Hyperlipidemia (\%) & 0,76 & $0,22-0,95$ & 0,08 & - & - & - & 1,37 & $1,01-1,67$ & 0,01 & 2,56 & $1,12-4,06$ & $<0,001$ \\
\hline Insulin (\%) & - & - & - & 0,21 & $0,1-0,43$ & 0,9 & 4,3 & $2,34-7,87$ & $<0,001$ & 4,71 & $2,3-9,65$ & $<0,001$ \\
\hline
\end{tabular}

BMI: body mass index; CAD: coronary artery disease; LDL-C: LDL cholesterol; TG: triglycerides.

\section{Ethical Approval}

All human studies have been approved by the appropriate ethics committee and have therefore been performed in accordance with the ethical standards laid down in the 1964 Declaration of Helsinki and its later amendments.

\section{Consent}

All persons gave their informed consent prior to their inclusion in the study.

\section{Conflict of Interests}

The authors declare that they have no conflict of interests.

\section{References}

[1] R. McPherson, A. Pertsemlidis, N. Kavaslar et al., "A common allele on chromosome 9 associated with coronary heart disease," Science, vol. 316, no. 5830, pp. 1488-1491, 2007.

[2] N. J. Samani, J. Erdmann, A. S. Hall et al., "Genomewide association analysis of coronary artery disease," The New England Journal of Medicine, vol. 357, no. 5, pp. 443-453, 2007.

[3] A. Helgadottir, G. Thorleifsson, A. Manolescu et al., "A common variant on chromosome 9p21 affects the risk of myocardial infarction," Science, vol. 316, no. 5830, pp. 1491-1493, 2007.

[4] T. L. Assimes, J. W. Knowles, A. Basu et al., "Susceptibility locus for clinical and subclinical coronary artery disease at chromosome 9p21 in the multi-ethnic ADVANCE Study," Human Molecular Genetics, vol. 17, no. 15, pp. 2320-2328, 2008.

[5] H. M. Broadbent, J. F. Peden, S. Lorkowski et al., "Susceptibility to coronary artery disease and diabetes is encoded by distinct, tightly linked SNPs in the ANRIL locus on chromosome 9p," Human Molecular Genetics, vol. 17, no. 6, pp. 806-814, 2008.

[6] E. Zeggini, M. N. Weedon, C. M. Lindgren et al., "Replication of genome-wide association signals in UK samples reveals risk loci for type 2 diabetes," Science, vol. 316, no. 5829, pp. 1336-1341, 2007.
[7] R. McPherson, "Chromosome 9p21 and coronary artery disease," The New England Journal of Medicine, vol. 362, no. 18, pp. 1736-1737, 2010.

[8] The Wellcome Trust Case Control Consortium, "Genome-wide association study of 14,000 cases of seven common diseases and 3,000 shared controls," Nature, vol. 447, pp. 661-678, 2007.

[9] G. E. Palomaki, S. Melillo, and L. A. Bradley, "Association between 9p21 genomic markers and heart disease: a metaanalysis," The Journal of the American Medical Association, vol. 303, no. 7, pp. 648-656, 2010.

[10] H. Schunkert, A. Götz, P. Braund et al., "Repeated replication and a prospective meta-analysis of the association between chromosome 9p21.3 and coronary artery disease," Circulation, vol. 117, no. 13, pp. 1675-1684, 2008.

[11] G. G. Gensini, "A more meaningful scoring system for determining the severity of coronary heart disease," The American Journal of Cardiology, vol. 51, no. 3, p. 606, 1983.

[12] G. Mancia, G. De Backer, A. Dominiczak et al., "Management of arterial hypertension of the European Society of Hypertension; European Society of Cardiology. Guidelines for the management of arterial hypertension: the Task Force for the Management of Arterial Hypertension of the European Society of Hypertension (ESH) and of the European Society of Cardiology (ESC)," Journal of Hypertension, vol. 25, pp. 11051187, 2007.

[13] J.-G. Wang, J. A. Staessen, S. S. Franklin, R. Fagard, and F. Gueyffier, "Systolic and diastolic blood pressure lowering as determinants of cardiovascular outcome," Hypertension, vol. 45, no. 5, pp. 907-913, 2005.

[14] S. Ye, J. Willeit, F. Kronenberg, Q. Xu, and S. Kiechl, "Association of genetic variation on chromosome 9p21 with susceptibility and progression of atherosclerosis: a populationbased, prospective study," Journal of the American College of Cardiology, vol. 52, no. 5, pp. 378-384, 2008.

[15] Z. Chen, Q. Qian, G. Ma et al., "A common variant on chromosome 9p21 affects the risk of early-onset coronary artery disease," Molecular Biology Reports, vol. 36, no. 5, pp. 889-893, 2009.

[16] W. Meng, A. E. Hughes, C. C. Patterson, C. Belton, F. Kee, and P. P. McKeown, "Chromosome 9p21.3 is associated with earlyonset coronary heart disease in the Irish population," Disease Markers, vol. 25, no. 2, pp. 81-85, 2008. 
[17] W. T. Friedewald, R. I. Levy, and D. S. Fredrickson, "Estimation of the concentration of low-density lipoprotein cholesterol in plasma, without use of the preparative ultracentrifuge," Clinical Chemistry, vol. 18, no. 6, pp. 499-502, 1972.

[18] S. A. Miller, D. D. Dykes, and H. F. Polesky, "A simple salting out procedure for extracting DNA from human nucleated cells," Nucleic Acids Research, vol. 16, no. 3, article 1215, 1988.

[19] S. Little, "Amplification-refractory mutation system (ARMS) analysis of point mutations," in Current Protocols in Human Genetics, A. L. Boyle, Ed., pp. 981-982, John Wiley \& Sons, New York, NY, USA, 2001.

[20] A. Doria, J. Wojcik, R. Xu et al., "Interaction between poor glycemic control and 9p21 locus on risk of coronary artery disease in type 2 diabetes," Journal of the American Medical Association, vol. 300, no. 20, pp. 2389-2397, 2008.

[21] N. V. Rivera, R. Carreras-Torres, R. Roncarati et al., "Assessment of the 9p21.3 locus in severity of coronary artery disease in the presence and absence of type 2 diabetes," BMC Medical Genetics, vol. 14, article 11, 2013. 


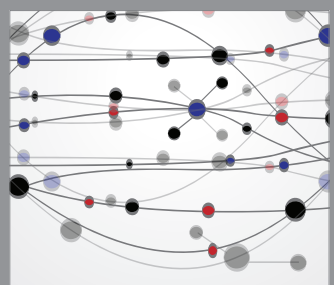

The Scientific World Journal
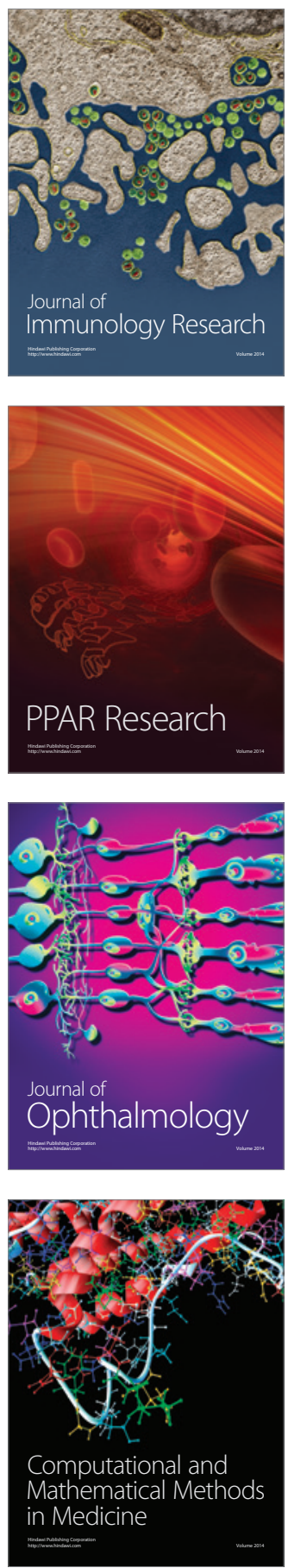

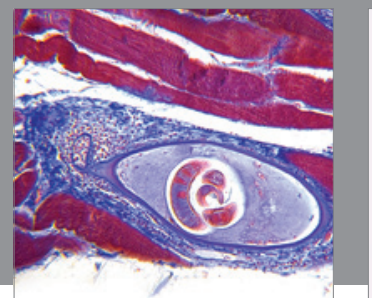

Gastroenterology

Research and Practice
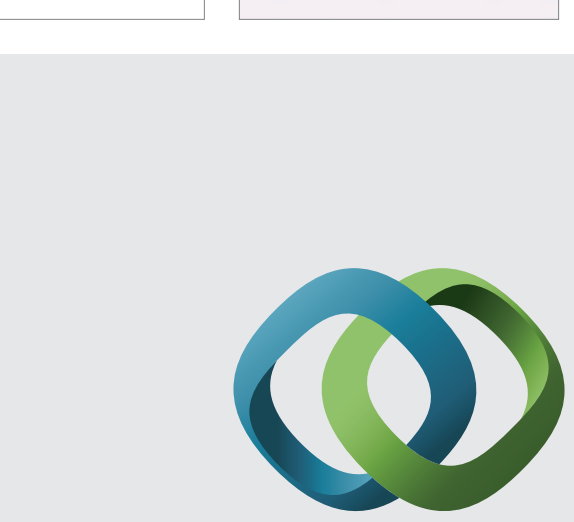

\section{Hindawi}

Submit your manuscripts at

http://www.hindawi.com
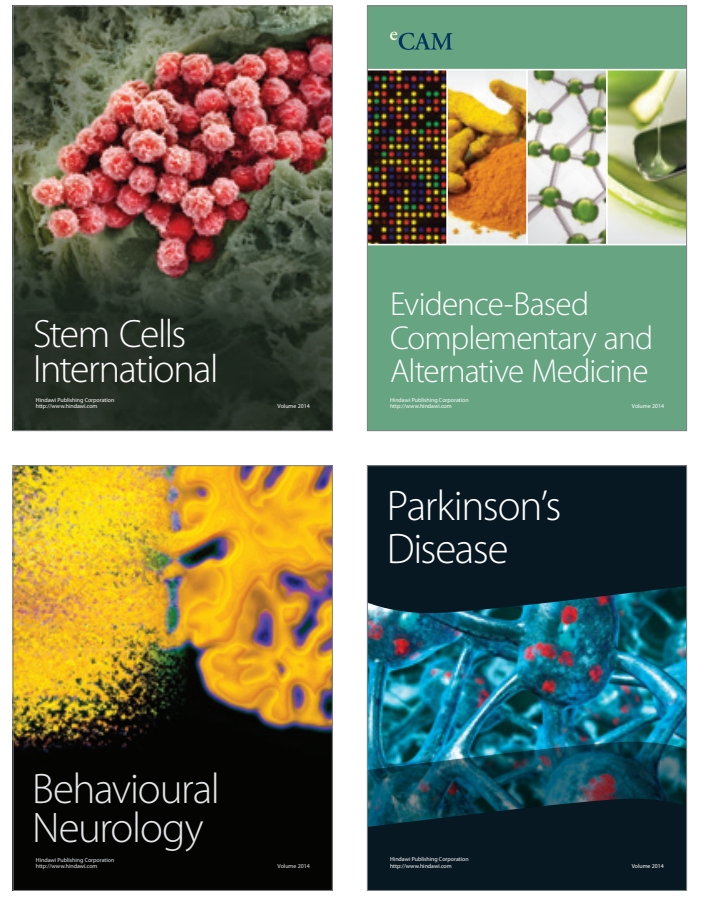
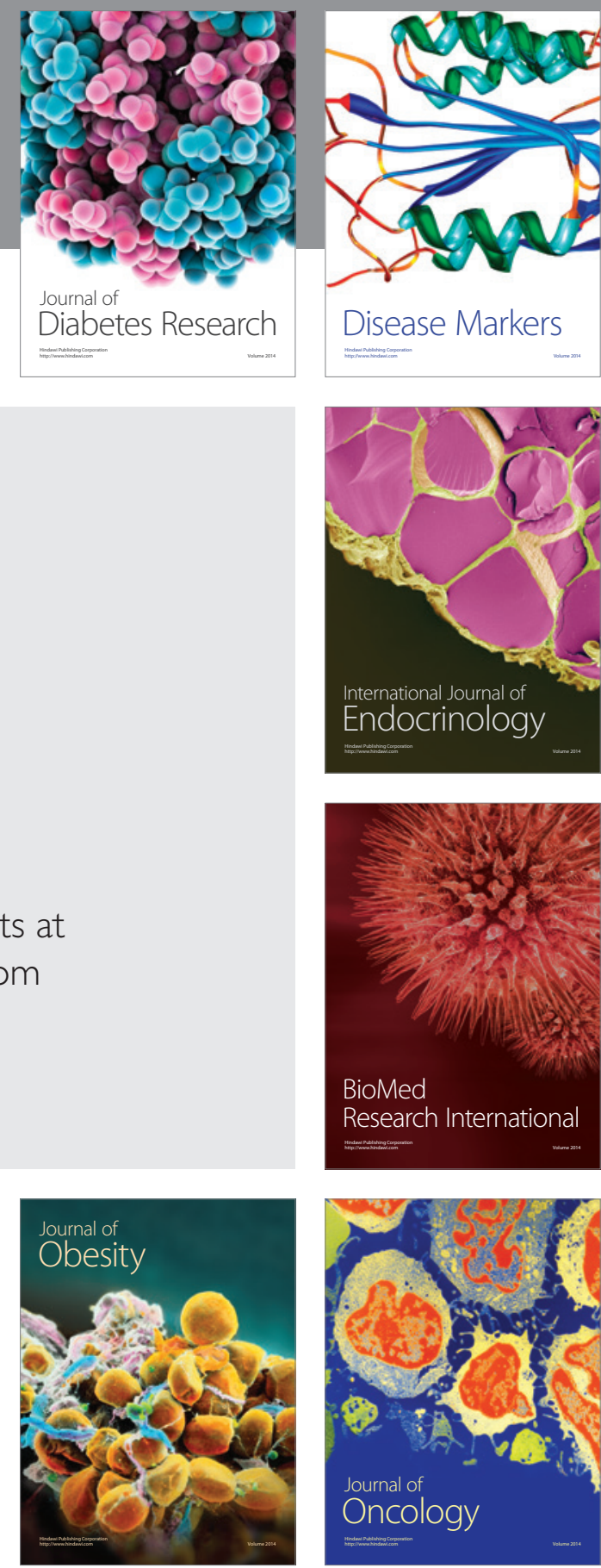

Disease Markers
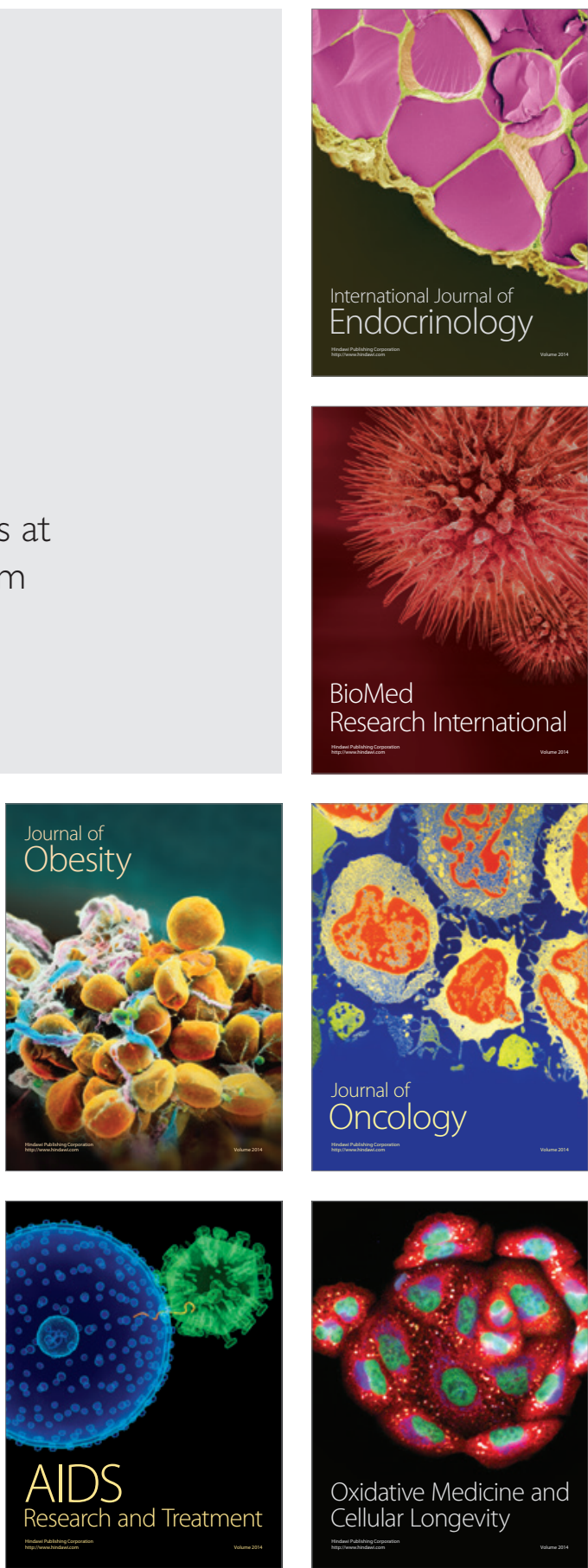